\title{
Cosmic Rays near the North Geomagnetic Pole in the Summers of 1955 and $1956^{*}$
}

\author{
H. V. NEHER \\ Norman Bridge Laboratory of Physics, California Institute of Technology, Pasadena, California
}

(Received March 27, 1957)

\begin{abstract}
The experiments herein described are a continuation of similar experiments performed in previous years near the north geomagnetic pole. In 1954 low-energy particles were found at high altitudes which had the characteristics of protons with energies down to $150 \mathrm{Mev}$. In 1955 flights to higher altitudes $\left(4 \mathrm{~g} \mathrm{~cm}^{-2}\right)$ indicated that these energies extended down to at least $100 \mathrm{Mev}$ for protons. The numbers of both the high-energy particles $(E>1 \mathrm{Bev})$ and those of low energy, in 1955 were less than in 1954. On one flight in 1955 the very low-energy particles were missing. In 1956, these low-energy particles ( $<300 \mathrm{Mev}$ for protons) were missing completely.

The "shape" of the ionization-depth curves at high altitudes appears to be a sensitive index of the presence or absence of these
\end{abstract}

low-energy particles. In 1956 the "shape" of the curves at low pressures $\left(<100 \mathrm{~g} \mathrm{~cm}^{-2}\right)$ was similar to those of 1951 when the low-energy radiation was missing and when a "knee" was present in the latitude curve at high altitudes. We infer that a "knee" was also present in 1956 but not in 1955 .

The fluctuations were also large in 1955 and 1956 compared with 1954. The average intensity of cosmic rays since 1954 has been decreasing with time as the sun approaches its maximum activity. This is in accordance with Forbush's relationship. However, in 1956 three of six flights showed intensities in the lower part of the atmosphere even less than any of the flights of 1937 at Saskatoon, Canada, in spite of the fact that the sun's activity in 1956 was less than in 1937.

\section{INTRODUCTION}

$\mathrm{P}^{\mathrm{R}}$ REVIOUS $^{1,2}$ data at high altitudes and high latitudes have been consistent with the general relationship stated by Forbush, ${ }^{3}$ that there is an inverse relationship between solar activity and cosmic-ray intensity. A further relationship, borne out by these and other experiments is that the fluctuations in the primaries from day to day are related directly to solar activity. In addition, the previous balloon flights have shown that the "knee" of the latitude curve at high altitudes is also dependent on solar activity and was missing in 1954 when the sun was very quiet although it was present in 1951.

The "shape" of the ionization-depth curve at high altitudes is quite dependent on the relative numbers of low- and high-energy particles striking the atmosphere. The change in shape from 1951 to 1954 was consistent with the change of ionization with latitude found from the simultaneous flights made in those two years using Bismarck, North Dakota as a base station. It appears, therefore, that one may predict with some certainty whether or not a "knee" is present by the "shape" of the ionization-depth curves taken near the geomagnetic poles for the very particles that so drastically change the shape of the curve at high altitudes are just those whose presence or absence cause the absence or presence of the "knee."

It was to gain further information on these low-energy particles that the balloon flights described herein were made in the summers of 1955 and 1956.

\section{APPARATUS}

The ionization chambers used in these experiments were as nearly as possible identical with those used in

* Assisted by the joint program of the Office of Naval Research and the U. S. Atomic Energy Commission.

1 Neher, Peterson, and Stern, Phys. Rev. 90, 655 (1953).

${ }^{2}$ H. V. Neher, Phys. Rev. 103, 228 (1956)

${ }^{3}$ S. E. Forbush, J. Geophys. Research 59, 525 (1954).
1954. ${ }^{2}$ To make sure that instrumental difficulties were minimized, great care was used in testing each instrument. The testing on the ion chambers began some time before the instruments were used, and a number of runs was made with each, and the consistency of operation was noted. It is noteworthy that of those instruments taken on the last three expeditions none has been rejected because of failure to give consistent results. Further, none of the instruments has been damaged by shipment even though some of the boxes have been partly broken open due to rough handling.

On two different days at Thule, Greenland in 1955, two instruments were carried up by the same balloon. Separate receiving antennas, receivers, and recorders were used for each instrument. One of the two ionization chambers contained argon at 8 atmospheres pressure while the other had 1 atmosphere of argon. The two were compared with three of our standard chambers before the flight, using the gamma rays from thorium $C^{\prime \prime}$. The results from the two instruments for the two flights are given in Fig. 1. Although the reasons for the very close agreement between the two ionization chambers with the different pressures is complicated somewhat by the different wall effects, it is quite clear that there is no indication of any appreciable lack of saturation of ion collection at the higher argon pressure at high altitudes where heavily ionizing particles are expected and hence where some recombination of ions might be expected if any was apt to be present.

Recently, Johnston ${ }^{4}$ has redetermined the absolute value of the ionization scale, which we have used for many years. Using a method similar to that employed by Millikan, ${ }^{5}$ the ionization produced by hard gamma rays was measured by balancing the charge so produced with the induced charge on an accurately known capacitance. Four of our standard ionization chambers were

${ }^{4}$ Alan R. Johnston, thesis, California Institute of Technology, 1956 (unpublished).

5 R. A. Millikan, Phys. Rev. 39, 397 (1932). 
then compared with the similar chamber in which the absolute value of the ionization was determined. By such means it was found that the scale of ionization we have used up to now differs from that determined by Johnston by only $0.3 \%$.

It should be noted, however, that in reducing to one atmosphere of air, such an atmosphere is reckoned in terms of $740 \mathrm{~mm}$ of $\mathrm{Hg}$ and $20^{\circ} \mathrm{C} .5,6$

The barometer and thermometer units were also checked before the flight. The barometer unit ${ }^{2,7}$ uses no mechanical magnification and has no detectable effects due to friction. It is believed that at the highest altitudes, the pressures are known to better than \pm 1 $\mathrm{mm}$ of $\mathrm{Hg}$. The temperature of the instrument is controlled during the flight to changes of only a few degrees centigrade as described elsewhere. ${ }^{2}$

\section{EXPERIMENTAL RESULTS}

Attention has already been called to the data from two identical instruments, except for the pressure of argon in the ion chambers, sent up with the same balloon. The two curves of Fig. 1 stay separated by about the same percentage irrespective of atmospheric depth, indicating that the added radiation on August 10, 1955 over August 7 was quite penetrating and hence

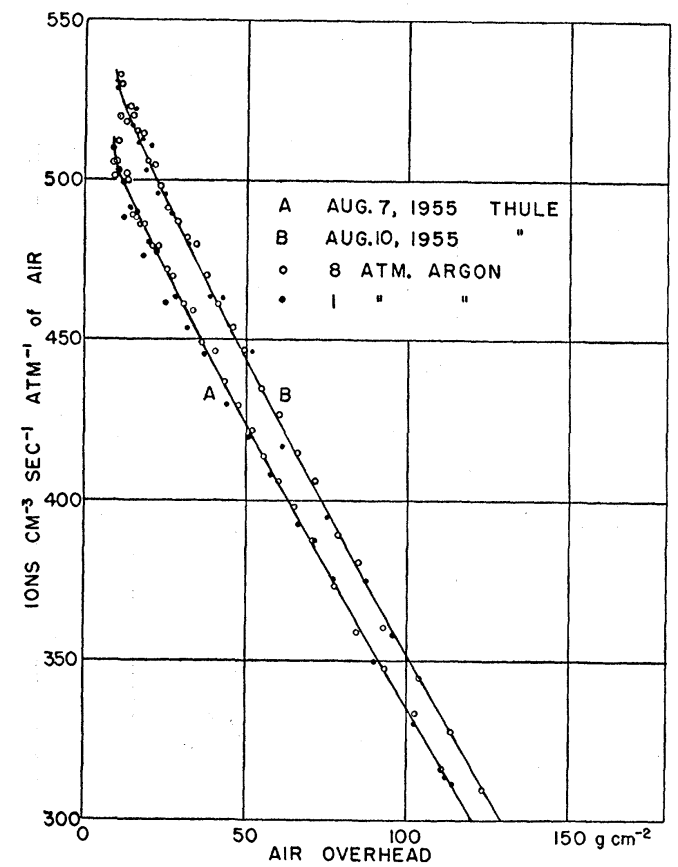

Fig. 1. On two different flights in 1955, two instruments were sent up with the same balloon. Ionization during the flight was calculated from comparison of each instrument with standard ion chambers on the ground before the flight, using $\gamma$ rays. There is very good agreement between the two chambers, one having 8 atmospheres of argon inside and the other 1 atmosphere. The difference between curves $A$ and $B$ represents a real change in cosmic rays from August 7 to August 10.

${ }^{6}$ R. A. Millikan, Phys. Rev. 37, 242 (1931).

${ }^{7}$ H. V. Neher, Rev. Sci. Instr. 24, 97 (1953).

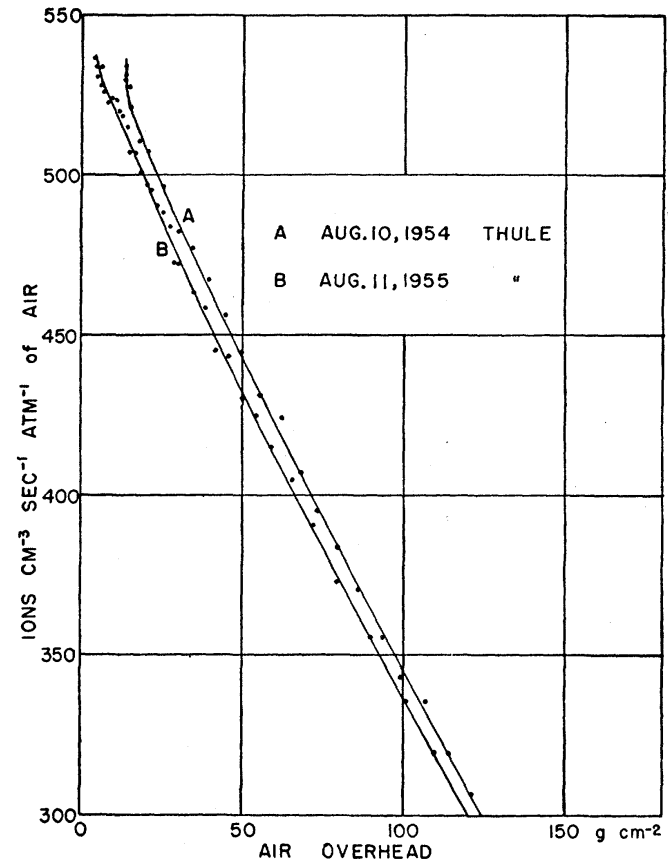

FIG. 2. The flight of August 11, 1955 went to $3.5 \mathrm{~g} \mathrm{~cm}^{-2}$. The turn-up of the curve occurred at a lower pressure than in 1954. If this rapid increase of ionization with decrease in pressure is due to protons (as seems likely), they have energies down to at least $100 \mathrm{Mev}$.

represents a change in relatively high-energy particles. For protons the energies would be several Bev. There was little change in the low-energy component that caused the turn-up of the curves at the lowest pressures. These low-energy particles have ranges of 10 to $15 \mathrm{~g} \mathrm{~cm}^{-2}$ and hence had energies down to $100 \mathrm{Mev}$.

On August 11, 1955 the turn-up of the curve did not occur until a pressure of about $8 \mathrm{~g} \mathrm{~cm}^{-2}$ was reached, as shown in Fig. 2. This is to be contrasted with August 10,1955 when the turn-up commenced at 11 $\mathrm{g} \mathrm{cm}^{-2}$ and on August 10, 1954 when it started at $15 \mathrm{~g} \mathrm{~cm}^{-2}$.

Other changes in the composition of the primary radiation from one day to another are brought out in Fig. 3. Here August 9, 1955 is compared with August 7, 1955 and August 10, 1954. The August 9 flight shows no turn-up at the lowest pressures, indicating that the low-energy particles responsible for the rapid increase in ionization on the previous day, were missing. Not only were the low-energy particles missing, but more highenergy particles were present than on the previous day as evidenced by the fact that curve $B$ lies above $A$ at the higher pressures.

An unusual situation occurred during this flight of August 9, 1955. It was normal until a pressure of about $120 \mathrm{~g} \mathrm{~cm}^{-2}$ was reached. From that pressure until about $75 \mathrm{~g} \mathrm{~cm}^{-2}$, quite violent fluctuations took place, and the average value of the ionization was higher than expected as may be seen from the points for curve $B$, 


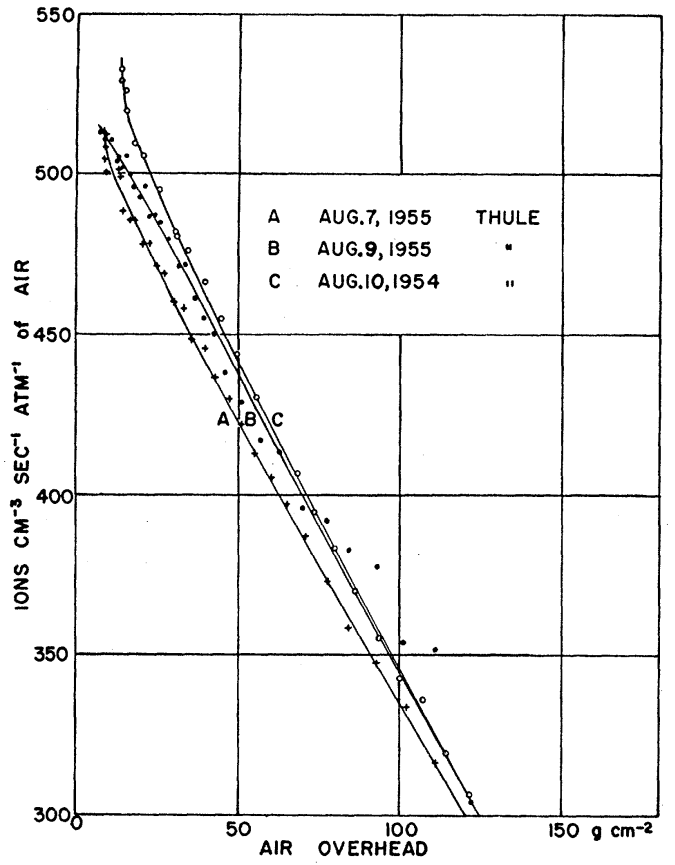

Fig. 3. On August 9 there were more high-energy particles and fewer of those of low energy than on August 7, 1955.

Fig. 3. At pressures of 60 to $40 \mathrm{~g} \mathrm{~cm}^{-2}$ the average ionization was less than expected. At still lower pressures the ionization again became consistent. This kind of behavior has never before been noticed on some 60 flights that have been made. We accredit this behavior to fluctuations in the primaries while the flight was taking place.

In the summer of 1956 , the characteristics of the radiation near the north geomagnetic pole were quite different. While the average ionization for the 6 flights of 1955 was only $2 \%$ less at high altitudes than in 1954 , the average for 6 flights in the summer of 1956 was $18 \%$ less than in 1954 at $25 \mathrm{~g} \mathrm{~cm}^{-2}$.

TABLE I. Comparison of balloon flights with ionization chambers in different years. Ionization is given in ions $\mathrm{cm}^{-3} \mathrm{sec}^{-1} \mathrm{atmos}^{-1}$ of air. Greenwich times are given for the maximum altitude.

\begin{tabular}{|c|c|c|c|c|c|c|}
\hline Location & $\left.\underset{\text { (Greenwich) }}{\text { Time }} \underset{\left(\mathrm{g} \mathrm{cm}^{-2}\right)}{\text { Atmos. depth }}\right\}$ & 10 & & $\begin{array}{c}40 \\
\text { nizatio }\end{array}$ & $n^{80}$ & 160 \\
\hline Saskatoon & Aug. $14-16,1937$ & $\ldots$ & 355 & 361 & 329 & 225 \\
\hline Baffin Bay & Aug. $\quad 4,1937$ & $\cdots$ & 363 & 367 & 328 & 22 \\
\hline \multirow{5}{*}{ Thule } & 16:52 July 25,1955 & $\ldots$ & & $\ldots$ & 388 & 247 \\
\hline & 19:51 Aug. 7, 1955 & 502 & 480 & 442 & 369 & 23 \\
\hline & Aug. 9,1 & 508 & 494 & 457 & 382 & 24 \\
\hline & Aug. 10, & 529 & 502 & 461 & 388 & 255 \\
\hline & 3 Aug. 11, 1 & 523 & 496 & 451 & 373 & 24 \\
\hline \multirow[t]{6}{*}{ Thule } & July 25,1 & 437 & 425 & 400 & 344 & 225 \\
\hline & 16:46 July 27,1956 & $\cdots$ & 407 & 386 & 332 & 217 \\
\hline & July 28,1 & $\cdots$ & $\cdots$ & 369 & 319 & 21 \\
\hline & 16:31 July 31,1 & $\ldots$ & $\cdots$ & 386 & 332 & 21 \\
\hline & Aug. 2, 1956 & 426 & 416 & 394 & 339 & \\
\hline & 3 Aug. 5, 1956 & $\ldots$ & $\cdots$ & 392 & 337 & 224 \\
\hline
\end{tabular}

a This flight was made by Carmichael and Dymond at $85^{\circ}$ geomagnetic north and has been normalized to our flights as explained in reference 2 .
Solar activity was increasing in the summer of 1956 from its very low minimum of 1954 . A maximum of activity is expected in 1958. Hence, this trend agrees with that to be expected from the inverse relationship between solar activity and cosmic-ray intensity as stated by Forbush. ${ }^{3}$

To compare quantitatively the balloon ionization data taken in different years, Table I has been prepared. Here the ionization at certain specified pressures is given. In Fig. 4, four of the 1956 flights are plotted together with the flight of Carmichael and Dymond ${ }^{8}$ near Thule, Greenland made at the peak of solar activity in 1937. As explained previously, ${ }^{2}$ this latter curve has been normalized to our own flights at Saskatoon, Canada made close to the same time. The two curves are, in fact, very similar, departing from each other only near their maxima, as shown in Table I.

The comparison of the 1937 flight with those of 1956 shows more low-energy particles and fewer of those of higher energy in 1956 as against 1937. If the low-energy particles responsible for the change of "shape" of the curves at high altitudes are protons, as seems likely from the 1954 series of flights, ${ }^{2}$ then we conclude that their energy distribution not only fluctuates from day to day but from year to year.

To bring out the large changes that have taken place from 1954 to 1955 to 1956 , one flight from each of the three years is given in Fig. 5. The various flights of

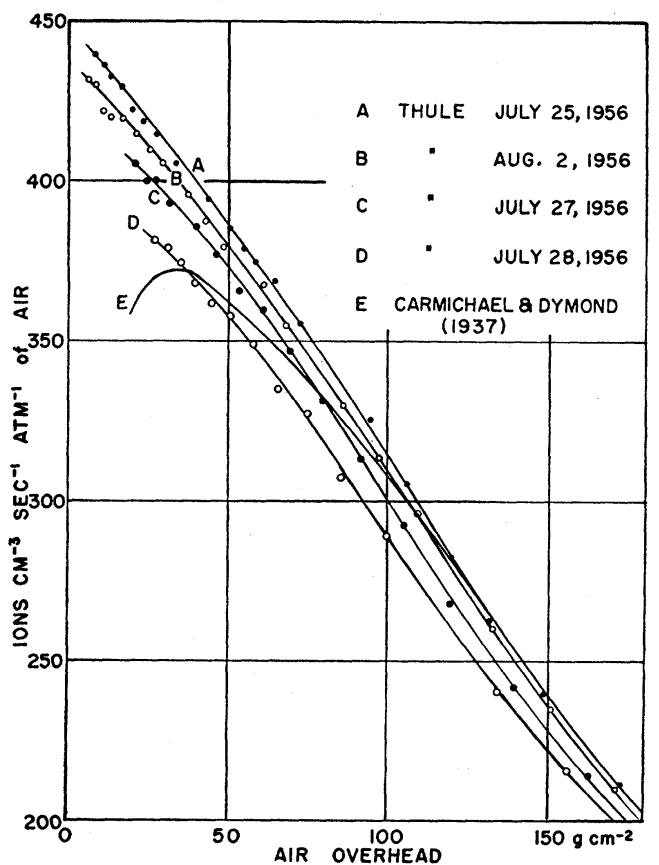

FIG. 4. The intensity was less at low altitudes and greater at high altitudes in 1956 than on Carmichael and Dymond's flight of 1937. We assume that this represents a real change in the energy distribution of the primaries.

${ }^{8} \mathrm{H}$. Carmichael and E. G. Dymond, Proc. Roy. Soc. (London) A171, 521 (1939). 
1954 near the geomagnetic pole were all very similar, and August 10 is chosen as representative. In 1955, August 7 is selected, while in 1956, July 28 is chosen. It remains to be seen how similar curves will appear in 1957 and 1958.

The presence or absence of the "knee" of the latitude curve for high altitudes depends on the absence or presence, respectively, of low-rigidity particles. Again we take the evidence of the flights of 1954 that these particles are protons. ${ }^{2}$ These are also the particles that so radically change the shape of the ionization depth curve at high altitudes. The evidence is, that in 1937, when the curves at Thule passed through a maximum at about $30 \mathrm{~g} \mathrm{~cm}^{-2}$, a "knee" existed. In 1951 there was no maximum, but there did exist an inflexion at about $60 \mathrm{~g} \mathrm{~cm}^{-2}$. Flights at various latitudes revealed a definite "knee" at that time. In 1954 no inflexion occurred at Thule, and no "knee" was present from flights at various latitudes. In 1955 there was no inflexion except on one day. Hence, we infer that, excluding this day, no "knee" existed. In 1956 there was an inflexion in the curves for all flights at Thule. The shape of the curves was very similar to those of 1951, as is illustrated by Fig. 6. We infer, therefore, that a "knee" also was present in 1956.

\section{DISCUSSION}

If one assumes that the fluctuations (excluding the solar flare increases) in the cosmic radiation incident on the earth are due to a modulation on the otherwise constant primaries which come from beyond the solar

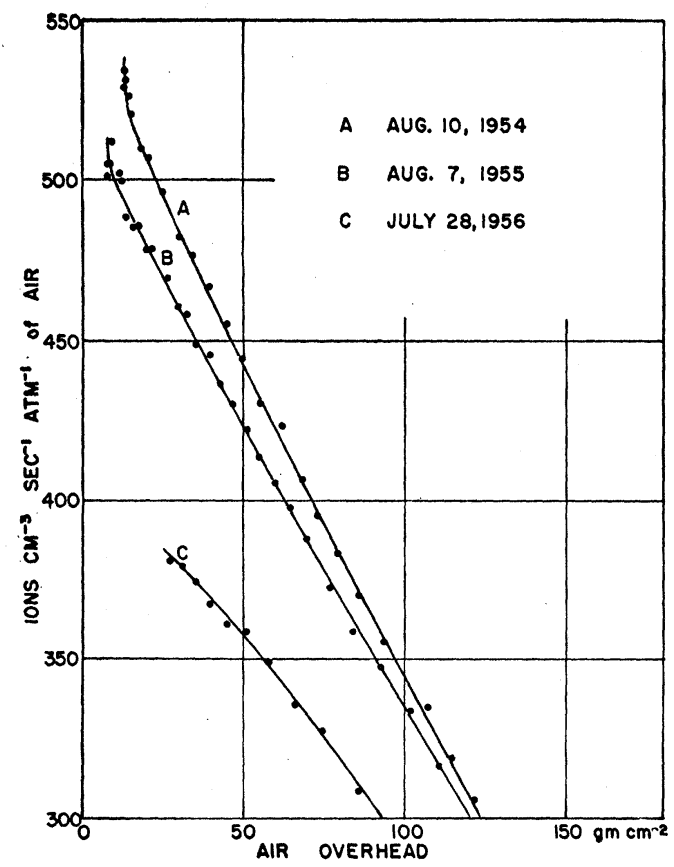

FIG. 5. Three flights have been selected from the years 1954, 1955, and 1956 at Thule to show the large changes that have taken place.

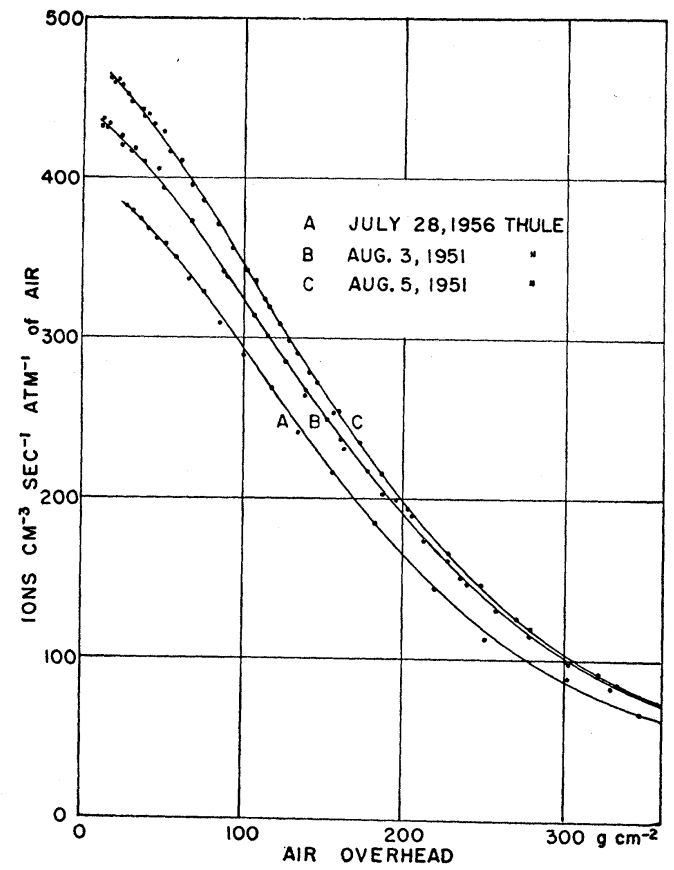

FIG. 6. The shape of the curves in 1956 was very similar to those taken in 1951. Since the presence of low-energy particles causes the turn-up of the curves at low pressures, there were no more of these particles present in 1956 than in 1951 when a "knee" existed in the latitude curve at high altitudes. We therefore assume that in 1956 a "knee" also existed. By the same token the "knee" was not present in 1955. (See Figs. 1 and 2.)

system, the present experiments point to some of the properties such a mechanism must have. Some of these have been listed before, ${ }^{1-3}$ but in the light of the present experiments, will be restated:

1. There is, in general, an inverse relationship between solar activity and cosmic-ray intensity.

2. There is a direct relationship between solar activity and the fluctuations in cosmic rays.

3. In general, particles of all energies, at least up to 30 or $40 \mathrm{Bev}$, are involved in the fluctuations. Those of low energy are, as a rule, affected much more than those of high energy.

4. While the relationship (3) is usually true, there are occasions when changes occur in the low-energy particles that are not accompanied by corresponding changes in those of high energy. In fact, the changes in the two components from one time to another may be of the opposite sign.

5. Whatever suppresses the radiation during a period around a solar maximum, must, during this period, be present continuously. At least in no case, at such a time, has the intensity jumped back to the value it had during a solar minimum.

6. Even when the lowest-energy particles, i.e., protons down to 100 to $150 \mathrm{Mev}$, can reach the earth, as in the summer of 1954 when solar activity was at a minimum, there may be increases in the high-energy 
particles $(>2-4 \mathrm{Bev})$ of at least $10 \%$ of the non-solarflare type. ${ }^{2}$

7. The changes in the primaries are usually worldwide, but there are exceptions when changes at Thule have not been accompanied by corresponding changes at other stations., ${ }^{1,2}$

It is evident that if one modulating mechanism is to satisfy all the requirements set forth above, it must act in a different and very complicated way at certain times. It appears, however, that it is only in the study of the nature of the changes in cosmic rays and the related geophysical, solar, and other associated phenomena that an understanding of the origin of the changes is to be found.

The alternative suggestion, that part or most of the low-energy radiation is of solar origin, has some attractive features. Even though emitted from the sun, the reflecting shell, assumed by Meyer, Parker, and Simpson $^{9}$ to be present to explain the isotropic distribution of the February 23, 1956 flare particles over the earth, would mix up the directions of arrival so that little if any diurnal effect would be evident. The following arguments may be made against the suggestion of solar origin: (1) There is a $180^{\circ}$ phase shift between solar activity and cosmic-ray intensity during a solar cycle. This is difficult to understand if conditions that give rise to high-energy particles from the sun are more likely to be present when the sun is active. (2) In no case would one expect the very highest energy particles to originate in or near the sun. If the low-energy part of the spectrum is of solar origin, then it would not be expected that the two energy distributions would join together in a smooth fashion as present experimental evidence seems to indicate. (3) Since the three elements, lithium, beryllium, and boron are present in the sun in extremely small amounts, it is difficult to see how sufficient fragmentation of heavy nuclei could occur to give a ratio of these elements to carbon, nitrogen, and oxygen of approximately 1 as is found in cosmic-ray particles. $^{10}$

\section{SUMMARY}

While the data presented here permit conclusions of a qualitative nature only to be drawn and leaves unanswered many questions regarding the radiation that changes with time, it is quite evident that much is

\footnotetext{
${ }^{9}$ Meyer, Parker, and Simpson, Phys. Rev. 104, 768 (1956)
}

${ }_{10}$ Noon, Herz, and O'Brien, Nature 179, 91 (1957). taking place to alter the primaries not only during short intervals of time, but over a period of years as well. Without, however, knowing many of the details, a number of interesting conclusions may be drawn from such data particularly when the situation at one period of time is compared with that at another. Also the measured latitude effect at high altitudes and latitudes, combined with the rigidity and range requirements permit one to say that at least a majority of the particles that change are protons. Down to $150 \mathrm{Mev}$ for protons it has been shown ${ }^{2}$ that during a solar cycle the number of primaries changes by at least a factor of 2.5. The present experiments show that as we approach the coming solar maximum, cosmic rays were little changed in 1955 over 1954 (when a solar minimum existed) but had decreased markedly from 1955 to 1956 becoming even less in 1956 at middle altitudes than in 1937 when the sun was at a peak of activity. The ionization-depth curve did not, however, pass through a maximum in 1956 as it did in 1937.

The "shape" of the ionization-depth curve at high altitudes appears to be a good index of the relative numbers of low-energy particles present in the primaries, and it now seems safe to predict whether or not a "knee" exists in the latitude curve at high altitudes by making balloon flights with ionization chambers near the geomagnetic poles only.

A search for a correlation between specific solar activity and the behavior of cosmic rays for the days flights were made in 1955 and 1956, shows nothing of a detailed nature. The sun was quite active during both periods, with many plages, spots, and small flares occurring almost daily. If anything, the sun was more active during the 1955 period than in 1956. It therefore appears that the average cosmic-ray intensity is not as much dependent on visual solar activity as it is on some accumulative effect.

\section{ACKNOWLEDGMENTS}

The author wishes to take this opportunity to express his appreciation to the Office of Naval Research for making the arrangements necessary to carry out these experiments. He extends his thanks to the North East Air Command and the personnel at Thule Air Force Base for their help in making the flights and supplying the necessary helium. Finally, he wishes to thank Dr. Alan R. Johnston who helped with the experimental work on both expeditions. 